\title{
Downregulation of adult and neonatal Nav1.5 in the dorsal root ganglia and axon of peripheral sensory neurons of rats with spared nerve injury
}

\author{
JUN WANG ${ }^{1,2}$, SHAO-WU OU ${ }^{2}$, YUN-FEI BAI $^{3}$, YUN-JIE WANG $^{2}$, \\ ZHI-QING DAVID XU ${ }^{3-5}$ and GUO-MING LUAN ${ }^{1,4,6}$
}

\begin{abstract}
${ }^{1}$ Department of Neurosurgery, Beijing Sanbo Brain Hospital, Capital Medical University, Beijing 100093; ${ }^{2}$ Department of Neurosurgery, The First Hospital of China Medical University, Shenyang 110001; ${ }^{3}$ Beijing Key Laboratory of Neural Regeneration and Repair, Department of Neurobiology, Capital Medical University; ${ }^{4}$ Center of Epilepsy, Beijing Institute for Brain Disorders, Beijing 100069, P.R. China; ${ }^{5}$ Department of Neuroscience, Karolinska Institute, S-17177 Stockholm, Sweden; ${ }^{6}$ Beijing Key Laboratory in Epilepsy, Beijing 100093, P.R. China
\end{abstract}

Received March 25, 2016; Accepted January 18, 2018

DOI: $10.3892 / \mathrm{ijmm} .2018 .3446$

\begin{abstract}
Previous studies demonstrated that Nav1.5 splice variants, including Nav1.5a and Nav1.5c, were expressed in dorsal root ganglia (DRG) neurons. However, since nine Nav1.5 isoforms have been identified, whether other Nav1.5 splice variants, especially the neonatal Nav1.5 splice variant, express in the DRG neurons is still unknown. In this study, we systematically investigated the expression of adult and neonatal Nav1.5 isoforms in the DRG neurons and axon of peripheral sensory neurons of rats with spared nerve injury (SNI) by RT-PCR, DNA sequencing, restriction enzyme digestion, immunohistochemistry and immunofluorescence methods. The results demonstrated that both adult and neonatal Nav1.5 isoforms were expressed in the DRG neurons, but their expression ratio was $\sim 2.5: 1$. In SNI rat models, the expression of both adult and neonatal Nav1.5 decreased by approximately a half in both mRNA and protein levels. In contrast, the expression of protein kinase $\mathrm{C}$ (PKC)- $\gamma$, one of the negative modulators for sodium currents, increased by $\sim 1$-fold. Taken together, this study first confirmed the expression of both adult and neonatal Nav1.5 isoforms in the DRG neurons and axon of peripheral sensory neurons of rat, but their expression level decreased in pain models. The upregulation of PKC- $\gamma$ may directly or
\end{abstract}

Correspondence to: Dr Zhi-Qing David Xu, Beijing Key Laboratory of Neural Regeneration and Repair, Department of Neurobiology, Capital Medical University, Beijing 100069, P.R. China

E-mail: xuzhiqingccmu@hotmail.com

Dr Guo-Ming Luan, Department of Neurosurgery, Beijing Sanbo Brain Hospital, Capital Medical University, Beijing 100093, P.R. China E-mail: luangm33@163.com

Key words: sodium channel, Nav1.5, dorsal root ganglia neurons, pain model, Rat indirectly downregulate the expression of Nav1.5 isoforms in SNI rat models, which may further involve in the pathological process of neuropathic pain.

\section{Introduction}

Voltage-gated sodium channel, a transmembrane protein, plays a major role in the generation and propagation of action potential in sensory neurons, which is a critical determinant of electrical excitability for nociception $(1,2)$. The three dimensional structure of voltage-gated sodium channel is still unknown, but is supposed to compose a large, poreforming $\alpha$-subunit and two small $\beta$-auxiliary subunits (3). The $\alpha$-subunit is the core or major components of voltage-gated sodium channel, which can function independently (3-5). So far, nine distinct voltage-gated sodium channel $\alpha$-subunits, namely Nav1.1-Nav1.9, have been cloned from various mammalian tissues (4). Of the nine distinct $\alpha$-subunits, all were observed in adult dorsal root ganglia (DRG) neurons except for Nav1.4 which was expressed abundantly in skeletal muscle $(6,7)$. Voltage-gated sodium channel Nav1.5, a tetrodotoxin-resistant (TTX-R) sodium channel, was first cloned from the heart and has been considered as the cardiac sodium channel $(8,9)$. However, as a matter of fact, it could also be detected in various mammalian tissues other than the heart, such as the brain and DRG neurons (9-19).

Previous studies confirmed the expression of Nav1.5 mRNA, including Nav1.5a and Nav1.5c isoforms, in adult mouse and rat DRG neurons $(14,15)$. Furthermore, Nav1.5 current was supposed to underlie the 'third TTX-R sodium current' in rat small DRG neurons (13). So far, nine Nav1.5 splice variants have been discovered (Nav1.5a-f and E28B-D) and four of them (Nav1.5a, Nav1.5c, Nav1.5d and Nav1.5e) have been proved to generate functional sodium channels (9). Whether other Nav1.5 splice variants, especially the neonatal Nav1.5 isoform (Nav1.5e) which shows different functional properties compared with adult Nav1.5 (20), express in the DRG neurons 
is still unknown. In this study, we systematically investigated the expression of neonatal and adult Nav1.5 in the DRG and axon of peripheral sensory neurons of rats with spared nerve injury (SNI) by RT-PCR, DNA sequencing, restriction enzyme digestion methods. The expression level of Nav1.5, as well as the expression ratio of neonatal Nav1.5 versus adult Nav1.5, in the DRG neurons and axon of peripheral sensory neurons was explored in this study. The expression and distribution of total Nav1.5 protein in these structures was also detected by immunohistochemistry and immunofluorescence method. In addition, the expression of protein kinase $\mathrm{C}$ (PKC), the activation of which was supposed to modulate the biophysical properties of TTX-R INa, in the DRG neurons and axon of peripheral sensory neurons of SNI rats was also detected in this study.

\section{Materials and methods}

Animal models. Healthy male Sprague-Dawley rats at the age of 8-10 weeks and weighing 200-250 g were provided by the Animal Experimentation Center of Capital Medical University. They were housed two per group and maintained on a 12-h light/dark cycle with food and water available ad libitum under constant temperature $\left(23 \pm 2^{\circ} \mathrm{C}\right)$. The investigation was approved by the Ethics Committee of Animal Experimentation of Capital Medical University and conformed to the principles outlined in the NIH Guide for the Care and Use of Laboratory Animals (NIH Publications no. 80-23).

The SNI rat models were established according to the previous study (21). In conclusion, the SD rats were anesthetized with pentobarbital sodium $(0.06 \mathrm{~g} / \mathrm{kg})$ and the surgery was conducted at room temperature $\left(18-22^{\circ} \mathrm{C}\right)$. After exposing the sciatic nerve and its three terminal branches (the sural, common peroneal and tibial nerves) by incising the skin and separating the muscle, axotomy and ligation of the tibial and common peroneal nerves was performed, leaving the sural nerve intact. Then the peroneal and the tibial nerves were tight-ligated with 4-0 surgical silk and sectioned distal to the ligation, removing $5 \mathrm{~mm}$ of the distal nerve stump.

RNA isolation and RT-PCR. On day 14 after surgery, the DRG (L4-6) and axon of the peripheral sensory neurons was removed. Total RNA was extracted by using RNA out kit (Takara, Shiga, Japan) according to the manufacturer's instructions.cDNAs were generated from $1 \mu \mathrm{g}$ of total RNA using the reverse transcription polymerase chain reaction (RT-PCR) kit (Life Technologies, Carlsbad, CA, USA). The PCR primer used for amplification of adult and neonatal Nav1.5 was designated to target both exon 6 and 6A: forward, 5'-TTCTGCCTGCATGCATTCACCTT-3' and reverse primer, 5'-GCAGAAGACAGTGAGGACCA-3', with a product length of $240 \mathrm{bp}$. The PCR reaction conditions consisted of $95^{\circ} \mathrm{C}$ for $5 \mathrm{~min}$ followed by 36 cycles of $95^{\circ} \mathrm{C}$ for $30 \mathrm{sec}, 66^{\circ} \mathrm{C}$ for $30 \mathrm{sec}, 72^{\circ} \mathrm{C}$ for $30 \mathrm{sec}$ and final elongation at $72^{\circ} \mathrm{C}$ for $5 \mathrm{~min}$. Glyceraldehyde 3 -phosphate dehydrogenase (GAPDH) was used for reference gene: forward, 5'-GACCACCCAGCCCAGCAAGG-3' and reverse primer, 5'-TCCCCAGGCCCCTCCTGTTG-3', with a product length of $144 \mathrm{bp}$. The mRNA expression was analyzed with the gel analysis system, and the relative expression level of Nav1.5 was calculated by dividing the gray value of its band by the gray value of the GAPDH band.
DNA sequencing. PCR products were separated on $2 \%$ agarose gel by electrophoresis. The fragments of the expected size were extracted and purified by using gel extraction kit (Qiagen, Valencia, CA, USA) and then sequenced directly by using 3730x1 DNA sequencer (Applied Biosystem, Foster City, CA, USA).

Restriction enzyme digestion. Restriction enzyme SacI was used to digest the total PCR products in order to distinguish the neonatal Nav1.5 splice variant from the total Nav1.5 cDNAs. The reaction system was $30 \mu \mathrm{l}$ in total, containing $8 \mu \mathrm{l}$ PCR products, $0.5 \mu \mathrm{l}$ SacI enzyme, $3 \mu 1$ loading buffer and $18.5 \mu \mathrm{l}$ super-purified water. After $1 \mathrm{~h}$ incubation at $38^{\circ} \mathrm{C}$ or overnight at room temperature, the PCR products were seperated on $2 \%$ agarose gel by electrophoresis to detect the digestion results.

Immunohistochemistry assay. Fresh specimens of the DRG (L4-6) and axon of the peripheral sensory neurons were fixed with $1-4 \%$ paraformaldehyde to prepare the paraffin-embedded specimens in $4-\mu \mathrm{m}$ thick sections. The streptavidin-peroxidase (SP) immunohistochemical method was applied. Immunohistochemistry was performed according to manufacturer's instructions of Histostain-SP kit (Invitrogen, Carlsbad, CA, USA). Sections were incubated with primary Nav1.5 antibody (rabbit, 1:100; Alomone, Jerusalem, Israel), and then incubated with the second antibody (biotinylated goat anti-rabbit IgG, 1:500; Invitrogen). Phosphate-buffered saline (PBS) replaced the primary antibody to serve as the negative control and the slide with known positive Nav1.5 expression in human atrial muscle served as the positive control.

Western blot analysis. The antibodies used for western blot analysis were as follows: rabbit anti-Nav1.5 polyclonal antibody (1:200; Alomone); rabbit polyclonal anti-PKC- $\gamma$ antibody (1:1,000; Santa Cruz Biotechnology, Inc., Santa Cruz, CA, USA); rabbit anti- $\alpha$ tubulin monoclonal antibody $(1: 10,000$; Millipore, Billerica, MA, USA); horseradish peroxidaselinked anti-rabbit and anti-mouse IgG secondary antibody (1:2,000; Cell Signaling Technology, Danvers, MA, USA). Approximately $100 \mathrm{mg}$ of tissue was rinsed with precooled PBS $\left(4^{\circ} \mathrm{C}\right)$, mixed with $500 \mu 1$ of RIPA strong lysate buffer, homogenized with a homogenizer, and left for lysis at $4^{\circ} \mathrm{C}$ overnight. After centrifugation at $12,000 \mathrm{rpm}$ for $30 \mathrm{~min}$ at $4^{\circ} \mathrm{C}$, the supernatants were collected and assayed for protein content. The protein concentration was determined using the BCA method, $100 \mu \mathrm{g}$ of protein was loaded into each lane for a 6-10\% sodium dodecyl sulfate-polyacrylamide gel electrophoresis (SDS-PAGE) running at a constant voltage of $80 \mathrm{~V}$ for $2 \mathrm{~h}$. The proteins were then transferred onto a polyvinylidene fluoride (PVDF) membrane at a constant current of $400 \mathrm{~mA}$ for $2 \mathrm{~h}$. The membrane was blocked with $10 \%$ non-fat milk for $2 \mathrm{~h}$ and incubated with primary antibody overnight at $4^{\circ} \mathrm{C}$. Then the membrane was rinsed with TBST 3 times and incubated with horseradish peroxidase-labeled secondary antibody with shaking at room temperature for $1 \mathrm{~h}$, and finally exposed and developed using the ECL system. Immunoreactive protein bands were detected with an enhanced chemiluminescence reagent (ECL-Plus) and densitometrically quantitated according to the manufacturer's instructions (Amersham 

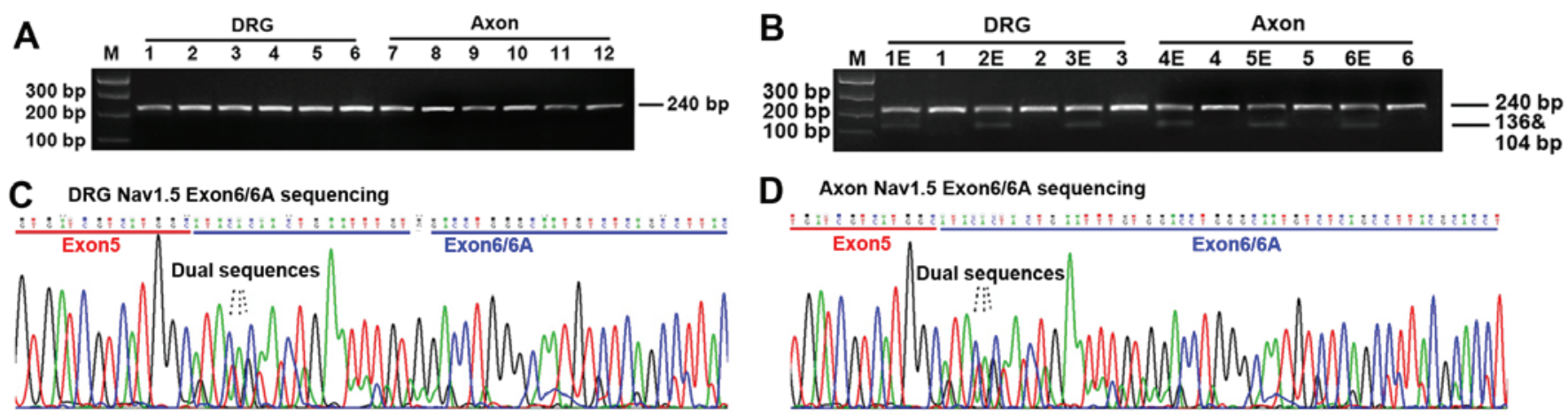

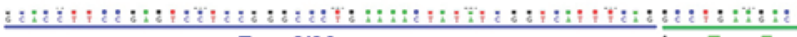
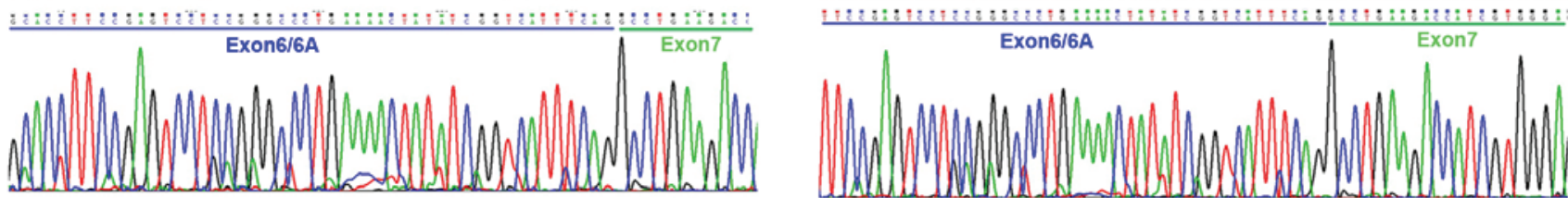

Figure 1. RT-PCR and direct DNA sequencing results. (A) RT-PCR results. Electrophoresis showed a single and clear band with the expected size (240 bp) on the agarose gel. M, Marker; 1-6, normal dorsal root ganglia (DRG) neurons; 7-12, normal axon of peripheral sensory neurons. (B) Digestion results by restriction enzyme SacI. 1E-6E, PCR products with restriction enzyme $S a c \mathrm{I}$; 1-6, PCR products without restriction enzyme $S a c \mathrm{I}$. As indicated by the electrophoresis results, PCR products with restriction enzyme SacI can generate two more bands on the agarose gel, with the expected size of 136 and 104 bp, respectively, indicating that the fragments including exon 6A were digested into another two fragments while that containing exon 6 was preserved. (C and D) DNA sequencing results. Direct DNA sequencing demonstrated that single sequence presented in the exon 5 or 7 coding region, but a dual sequence appeared in exon 6 coding region, indicating that both exon 6 and 6A were expressed in the DRG neurons and axon of peripheral sensory neurons.

Pharmacia Biotech, Piscataway, NJ, USA). The protein expression was analyzed with the gel analysis system, and the relative expression levels of targeted protein were calculated by dividing the gray value of its band by the gray value of the $\alpha$-tubulin band. The experiments were repeated 3 times.

\section{Results}

Both neonatal and adult Nav1.5 mRNA are expressed in the DRG neurons and axon of peripheral sensory neurons of adult rat. In order to detect the expression of neonatal Nav1.5 (Nav1.5e) isoform in the DRG neurons and axon of peripheral sensory neurons of rats, we used a special PCR primer targeting both exon 6 (adult Nav1.5) and exon 6A (neonatal Nav1.5) of SCN5A. Electrophoresis was made after PCR and the results demonstrated that a single band with the expected size (240 bp) appeared on the $2 \%$ agarose gel (Fig. 1). Direct DNA sequencing of the PCR products was performed after gel extraction. The results showed that a single sequence presented in exon 5 or 7 coding region, but a dual sequence appeared in exon 6 or $6 \mathrm{~A}$ coding region (Fig. 1). DNA sequence analysis revealed that both exon 6 and $6 \mathrm{~A}$ of SCN5A gene were inclusively expressed in the PCR products, indicating the expression of both adult and neonatal Nav1.5 channels (Nav1.5e) in the DRG neurons and axon of peripheral sensory neurons of adult rats.

In order to explore the expression quantification of the adult and neonatal Nav1.5 splice variants, we used restriction enzyme SacI to digest the PCR products for a specific restriction enzyme site of $S a c I$ was found in exon 6A rather than exon 6 of SCN5A gene. As indicated in Fig. 1, PCR fragments including exon 6A were digested into two bands while that containing exon 6 was preserved. Therefore, the relative amounts of digested and undigested PCR products represented the quantification of neonatal and adult Nav1.5 cDNA, respectively. The expression ratio of adult (wild Nav1.5) versus neonatal Nav1.5 (Nav1.5e) in the DRG neurons was $\sim 2.5: 1$ judged from the signal quantification of pre- and postdigestion by autoradiography (Fig. 1) $(n=6)$. Similar results were found in the axon of peripheral sensory neurons of adult rats (Fig. 1) $(\mathrm{n}=6)$.

The expression of Nav1.5 mRNA is decreased in the DRG neurons and axon of peripheral sensory neurons of rat with spared nerve injury. RT-PCR methods was used to detect the expression of neonatal and adult Nav1.5mRNA in the DRG neurons and axon of peripheral sensory neurons of rats with spared nerve injury. The results demonstrated the expression of total Nav1.5 mRNA (adult with neonatal Nav1.5) decreased by $\sim 55 \%$ in SNI rat models compared with that in controls, indicating a downregulation of Nav1.5mRNA expression in the neuropathic pain models. Further investigation was made in order to clarify whether the neonatal and adult Nav1.5 decreased simultaneously. As indicated in Figs. 2 and 3, restriction enzyme digestion method was used to figure out the quantification of neonatal and adult Nav1.5 mRNA expressed in the DRG neurons and axon of peripheral sensory neurons of rats with spared nerve injury. The results confirmed that both adult and neonatal Nav1.5 mRNA decreased in the DRG neurons of SNI rats, with the adult and neonatal Nav1.5 mRNA decreased by $\sim 53$ and $56 \%$ ( $n=12$ for SNI rats; $n=6$ for controls), respectively. Similar expression pattern of Nav1.5 mRNA was found in the axon of peripheral sensory neurons of SNI rats, with the adult and neonatal Nav1.5 mRNA decreased by $\sim 55$ and $57 \%$ ( $n=12$ for SNI rats; $n=6$ for controls), respectively.

Expression of total Nav1.5 protein in the DRG neurons and axon of peripheral sensory neurons of rats. In order to investigate whether Nav1.5 protein was expressed in the DRG 

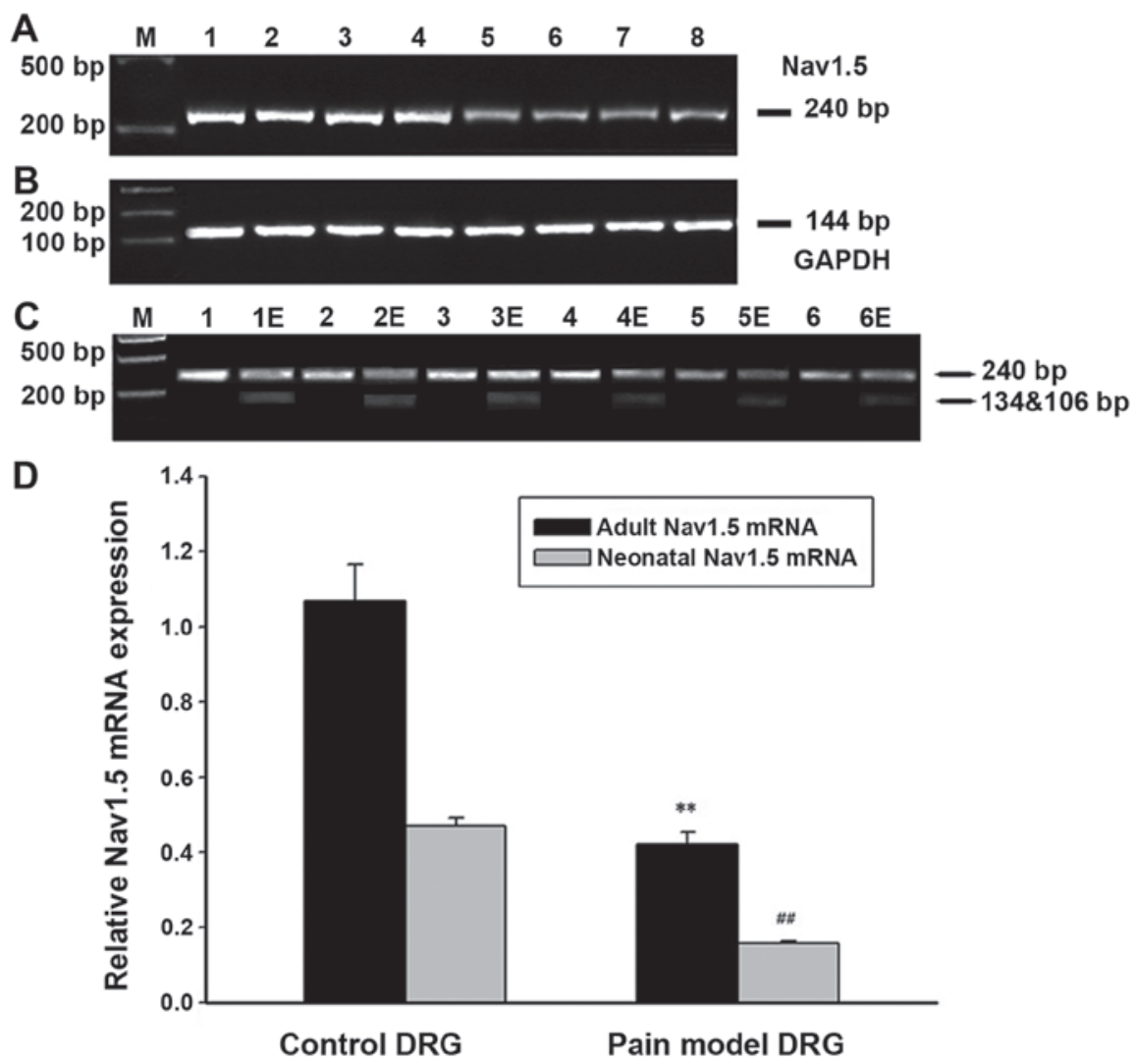

Figure 2. Expression of Nav1.5 mRNA in the dorsal root ganglia (DRG) neurons. (A) RT-PCR results in the DRG neurons and axon of peripheral sensory neurons of rats with spared nerve injury. (B) GAPDH was used as reference. (C) Digestion results by restriction enzyme SacI. (D) Statistical analysis of expression of both adult and neonatal Nav1.5 mRNA in the DRG neurons of neuropathic pain model of rats, with the adult and neonatal Nav1.5 mRNA decreased by $\sim 53$ and $56 \%$, respectively. Data are mean $\pm \mathrm{SEM} ;{ }^{*}, \mathrm{P}<0.05,[\mathrm{n}=12$ for spared nerve injury (SNI) rats; $\mathrm{n}=6$ for controls].

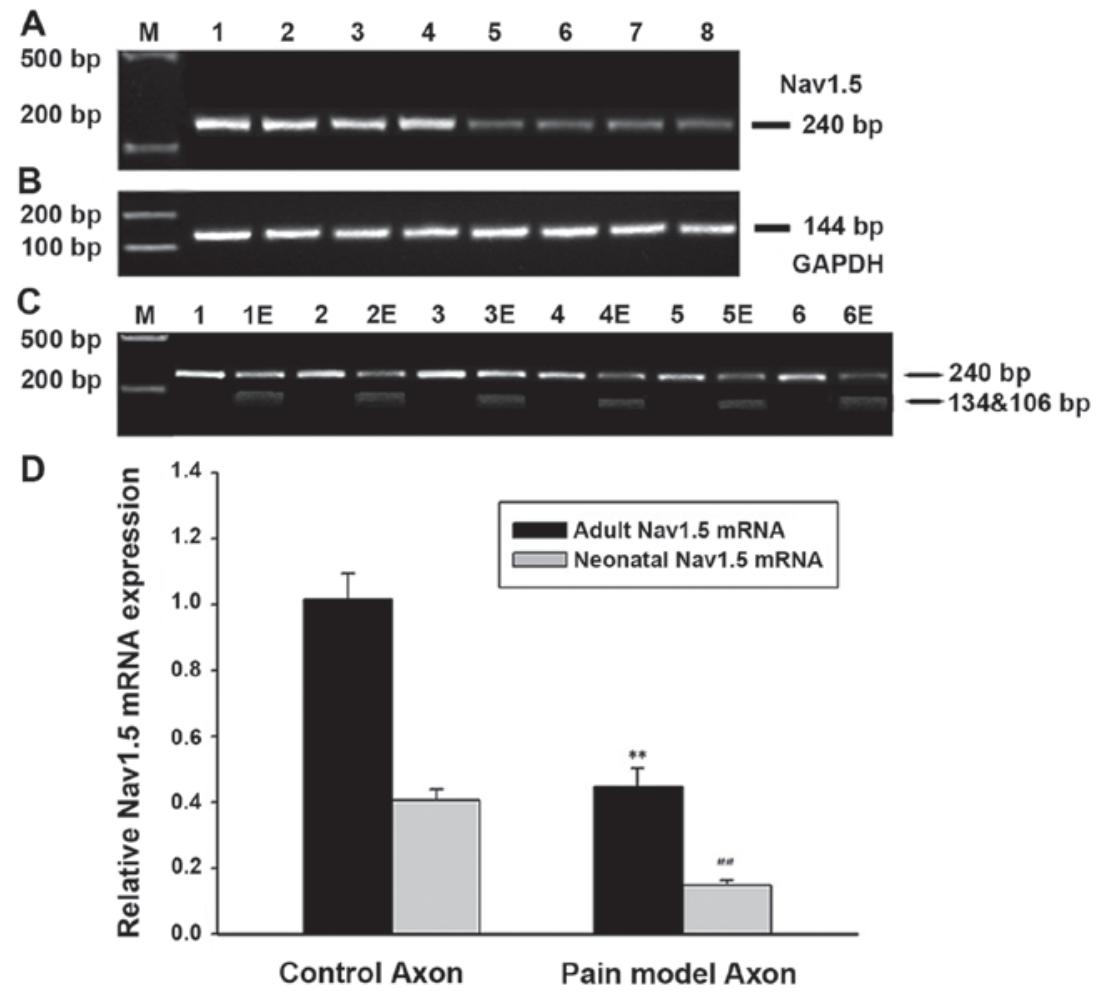

Figure 3. Expression of Nav1.5 mRNA in peripheral sensory neurons of rat with spared nerve injury. (A) RT-PCR results in the axon of peripheral sensory neurons of rats with spared nerve injury. (B) GAPDH was used as reference. (C) Digestion results by restriction enzyme SacI. (D) Statistical analysis of expression of both adult and neonatal Nav1.5 mRNA in the axon of peripheral sensory neurons of rat with spared nerve injury, with the adult and neonatal Nav1.5 mRNA decreased by $\sim 55$ and $57 \%$, respectively. Data are mean $\pm \mathrm{SEM} ;{ }^{*},{ }^{\prime} \mathrm{P}<0.01,[\mathrm{n}=12$ for spared nerve injury (SNI) rats; $\mathrm{n}=6$ for controls]. 

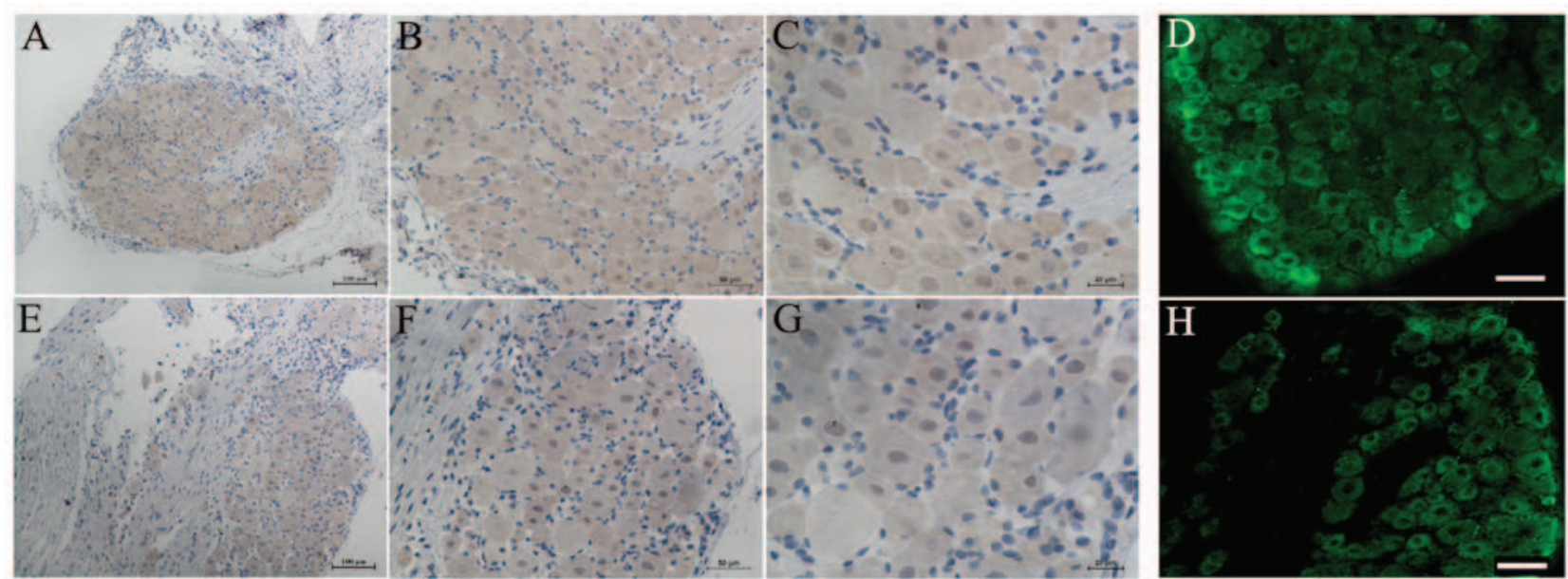

Figure 4. Immunohistochemistry and immunofluorescence results of Nav1.5 expression in the dorsal root ganglia (DRG) neurons of rats. (A-D) Normal DRG neurons. (D-F and H) DRG neurons of rats with spared nerve injury. Nav1.5 immunohistochemistry and immunofluorescence showed a decreased expression in the DRG neurons of rats with spared nerve injury compared with controls. Scale bars: (A and E) $100 \mu \mathrm{m}$; (B, D F and H) $50 \mu \mathrm{m}$; (C and G) $20 \mu \mathrm{m}$.

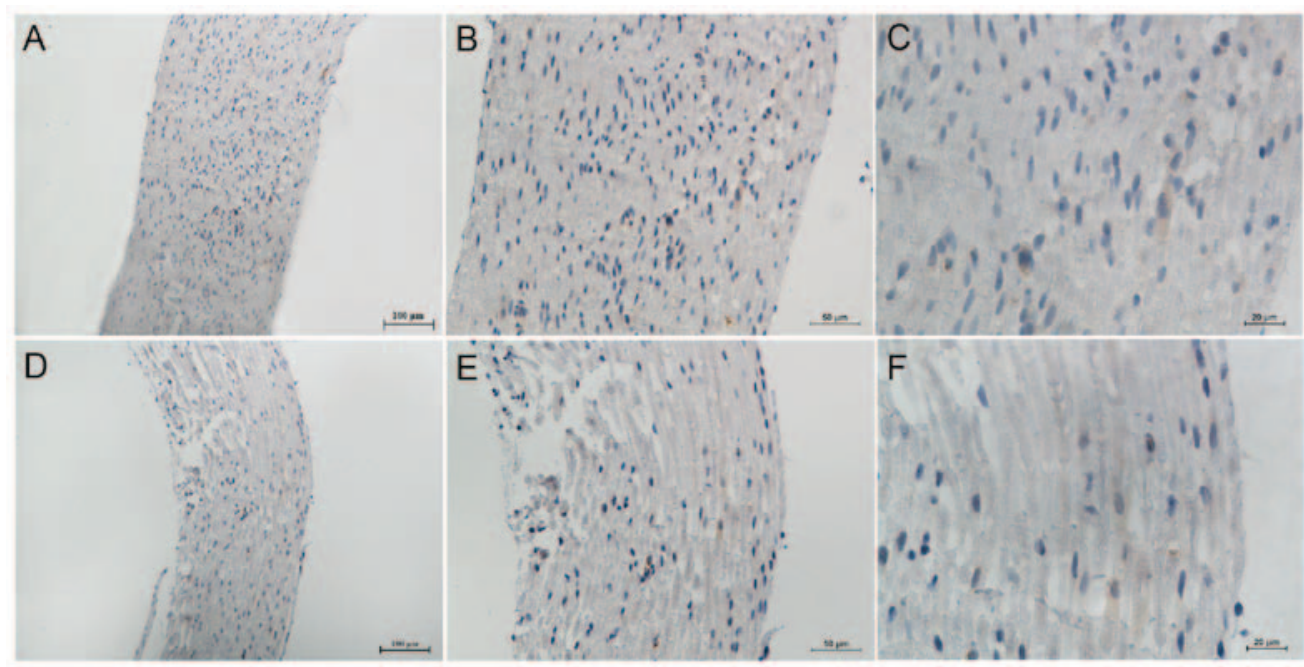

Figure 5. Immunohistochemistry results of Nav1.5 expression in the axon of peripheral sensory neurons. (A-C) Normal axon of peripheral sensory neurons. (D-F) Axon of peripheral sensory neurons of rats with spared nerve injury. Nav1.5 immunohistochemistry showed a decreased expression in axon of peripheral sensory neurons of rats with spared nerve injury compared with controls. Scale bars: $100 \mu \mathrm{m}(\mathrm{A}$ and D); $50 \mu \mathrm{m}$ (B and E); $20 \mu \mathrm{m}(\mathrm{C}$ and F).

neurons and axon of peripheral sensory neurons of rat, we used immunohistochemical and immunofluorescence methods to detect the expression and distribution of total Nav1.5 protein in these structures. The results demonstrated that Nav1.5 immunoreactivity could be detected in the membrane and plasma of DRG neurons and it showed stronger immunoreactivity in the small diameter sensory neurons compared with large ones (Fig. 4). Positive Nav1.5 staining results were also observed in the axon of peripheral sensory neurons, including the myelin sheath (Schwann cells) (Fig. 5). In general, the Nav1.5 immunoreactivity in the axon and myelin sheath was weaker than that in the DRG neurons. Similar to the RT-PCR results, the immunohistochemistry results of Nav1.5 also indicated a decreased expression of total Nav1.5 protein in the DRG neurons and axon of peripheral sensory neurons of SNI rats (Figs. 4 and 5).

Upregulation of PKC- $\gamma$ in the DRG neurons and axon of peripheral sensory neurons of rats with spared nerve injury.
The activation of PKC was supposed to modulate the biophysical properties of Nav1.5 channels, therefore, the expression of PKC- $\gamma$, which expressed abundantly in nervous system, was detected in the DRG neurons and axon of peripheral sensory neurons of rats with spared nerve injury by western blot method. The results demonstrated that the expression of PKC- $\gamma$ increased by $\sim 1$-fold in the DRG neurons of rats with spared nerve injury (Fig. $6, n=12$ for SNI rats; $n=6$ for controls). Similar expression pattern of PKC- $\gamma$ was found in the axon of peripheral sensory neurons of SNI rats (Fig. 7, $n=12$ for SNI rats; $n=6$ for controls). The results confirmed the upregulation of PKC $-\gamma$ in both the DRG neurons and axons of peripheral sensory neurons of pain model rats.

\section{Discussion}

Both adult and neonatal Nav1.5 are expressed in the DRG neurons and axons of peripheral sensory neurons. Previous studies have confirmed the expression and distribution of 

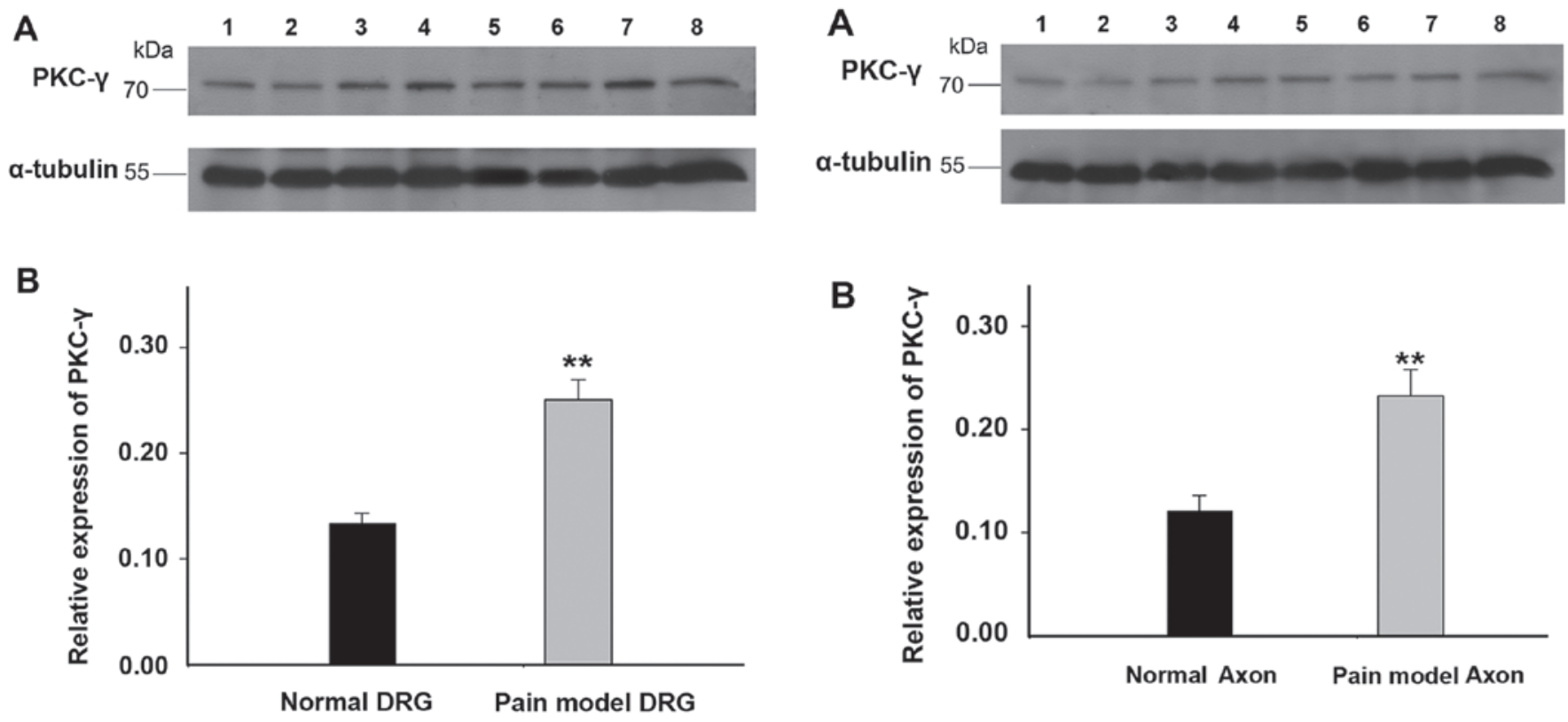

Figure 6. Western blot results of the expression of protein kinase C (PKC)- $\gamma$ in the dorsal root ganglia (DRG) neurons. (A) Expression of PKC- $\gamma$ in the DRG neurons of rats with spared nerve injury and controls. 1,2, normal DRG neurons; 3-8, DRG neurons of rats with spared nerve injury. $\alpha$-tublin was used as reference. (B) Statistical analysis of expression of Nav1.5 protein in the DRG of peripheral sensory neurons of rat with spared nerve injury compared with controls. Data are mean $\pm \mathrm{SEM} ;{ }^{*}, \mathrm{P}<0.01$, $[\mathrm{n}=12$ for spared nerve injury (SNI) rats; $\mathrm{n}=6$ for controls].

Nav1.5 in the adult and neonatal rat and mouse DRG neurons in both mRNA and protein levels (14). Moreover, the expression ratio of various Nav1.5 alternative splicing variants, including Nav1.5a and Nav1.5c, in the rat and mouse DRG neurons has also been investigated (15). These studies demonstrated that Nav1.5a was the major Nav1.5 isoform expressed in the DRG neurons and it showed different functional properties compared with full-length Nav.1.5 (wild Nav1.5) isoform $(14,15)$. Subsequently, Nav1.5 channel was also proved to produce the third TTX-R current in rat small DRG neurons by electrophysiological studies (13). However, since nine Nav1.5 splice variants have been discovered (Nav1.5a-f, E28B-D), whether other Nav1.5 isoforms are expressed in the DRG neurons is still unknown. To our knowledge, there was no report on the expression of neonatal Nav1.5 isoform in the DRG neurons. Therefore, in this study, we systematically investigated in the expression of neonatal Nav1.5 in the DRG neurons and the axons of peripheral sensory neurons of SNI rats. The results confirmed, for the first time, the expression of both neonatal and adult Nav1.5 isoforms in the DRG neurons and axon of peripheral sensory neurons.

The neonatal Nav1.5, also called Nav1.5e or nNav1.5, is characterized by an exon $6 \mathrm{~A}$ encoding for the $\mathrm{S} 3 / \mathrm{S} 4$ region in domain I (residues 205-234 in hNav1.5) $(22,23)$. Instead, the adult Nav1.5 is encoded by exon 6 of SCN5A. Both exon 6A and exon 6 have 92 nucleotides but differ at 31 nucleotide positions, resulting in 7 amino acid differences $(10,11,16,22,23)$. Notably, this alternative splicing from exon 6 to $6 \mathrm{~A}$ replaces a conserved negative aspartate residue in the adult Nav1.5 with a positive lysine (20). Thus, this amino acid change reversed the local charge, which may alter the electrophysiological properties

of Nav1.5. Subsequently, this hypothesis was confirmed by Onkal et al (20). As reported in their study, compared with the 'adult' Nav1.5 isoform, the 'neonatal' Nav1.5 channel exhibited i) a depolarized threshold of activation and voltage at which the current peaked; ii) much slower kinetics of activation and inactivation; iii) $50 \%$ greater transient charge (Nat) influx; iv) a stronger voltage dependence of time to peak; and v) a slower recovery from inactivation (20). Therefore, the alternative splicing of exon 6 and $6 \mathrm{~A}$ generated two electrophysiologically different Nav1.5 channels. In this study, the co-expression of neonatal and adult Nav1.5 in the DRG neurons and axons of peripheral sensory neurons was identified. However, the expression ratio of the two distinct Nav1.5 isoforms, which may affect the total Nav1.5 functional properties, in these structures was still uncertain. Therefore, we analyzed the difference between the sequence exon 6 and 6A. Occasionally, a specific restriction enzyme site of $S a c I$ was found in the exon $6 \mathrm{~A}$ rather than exon 6. Therefore, the restriction enzyme SacI was used to digest the PCR products in order to distinguish these two variants. The results demonstrated the expression ratio of adult (wild Nav1.5) versus neonatal Nav1.5 (Nav1.5e) in the DRG neurons and axon of peripheral sensory neurons was $2.5: 1$. Therefore, the results indicated that the Nav1.5 sodium current detected before was a compound product by both adult and neonatal Nav1.5 isoforms. Our findings provided a basic step for further investigation in the significance of the co-expression of adult and neonatal Nav1.5 in the DRG neurons.

Previous studies have detected the expression of Nav1.5 protein in the DRG neurons by western blot method (15). In this study, we used immunochemistry and immunofluorescence method to detect the expression of Nav1.5 protein in the DRG 
neurons and axon of peripheral sensory neurons. The results demonstrated that Nav1.5 immunoreactivity could be detected both in the DRG neurons and axon of peripheral sensory neurons, and it showed stronger immunoreactivity in the small diameter DRG neurons compared with large ones. These results, combined with the previous electrophysiological studies, confirmed the expression of Nav1.5 in the protein level. Although Nav1.5 was proved to express functionally in the DRG neurons, its specific role in the generation and propagation of action potential still remains unknown. Previous studies demonstrated that the functional properties of Nav1.5 in the DRG neurons was proved to be different from other TTX-R sodium channels, such as Nav1.8 and Nav1.9 channels, in the following aspects: half-time for activation, the time constant for inactivation, and the midpoints of activation and inactivation (13). Future studies should make deep investigation in clarifying the specific function of Nav1.5 in the DGR neurons and axons of peripheral sensory neurons.

Downregulation of adult and neonatal Nav1.5 in the DRG neurons and axons of peripheral sensory neurons of SNI model rats. Voltage-gated sodium channels in the DRG neurons and axons of peripheral sensory neurons are essential for the generation and propagation of action potential for nociception $(1,7,24,25)$. Since at least eight sodium channels have been detected to be expressed in the DRG neurons axons of peripheral sensory neurons $(6,7,25)$. It is important to clarify the specific contributions of various sodium channels to the generation and propagation of nociceptive information. Previous studies demonstrated that the expression of Nav1.5a and Nav1.5/Nav1.5c mRNA decreased by 48.1 and $46.4 \%$, respectively, in mouse lumbar L4 and L5 DRG neurons seven days after peripheral axotomy (15). In this study, we investigated the expression of neonatal and adult Nav1.5 mRNA and protein in the DRG neurons, as well as the axon of peripheral sensory neurons of rats with spared nerve injury. Similar to the expression of Nav1.5a and Nav1.5/Nav1.5c in the DRG neurons of peripheral nerve transection (axotomy) rat model, RT-PCR results demonstrated the expression of neonatal and adult Nav1.5 mRNA decreased approximately by half in the DRG neurons and axon of peripheral sensory neurons of rats with spared nerve injury. Immunochemistry and immunofluorescence results also indicated a downregulation of total Nav1.5 protein in these structures. In general, others and our findings confirmed the downregulation of Nav.5 channel in both mRNA and protein level in the neuropathic pain model.

The role of $P K C-\gamma$ in the modulation of sodium current and sodium channel gene expression in the DRG neurons. PKC- $\gamma$, one of the conventional PKC (cPKC) isozymes, is expressed abundantly in the nervous systems. Previous studies demonstrated that activation of PKC led to a reduction in the sodium current in both brain and heart (26-29). This effect was due to the phosphorylation of site $\operatorname{Ser}^{1503}$ in the Nav1.5 channel by activated PKC. Other studies further demonstrated that Nav1.5 channels stably expressed in human embryonic kidney cells were preferentially trafficked away from the plasma membrane by PKC activation, with a major contribution by $\mathrm{Ca}^{2+}$ sensitive or conventional PKC isoforms (30). Removal of the conserved PKC site $\mathrm{Ser}^{1503}$ eliminated the PKC-mediated effect to alter Nav1.5 channel trafficking (30). These results demonstrated
PKC may play a role to alter both the localization and functional properties of Nav1.5 channels. In this study, we investigated the expression of PKC- $\gamma$ and other PKC isoforms, including PKC $-\alpha$, PKC $-\delta$ and PKC- $\varepsilon$ in the DRG neurons and axons of peripheral sensory neurons of rats with spared nerve injury. Of these isoforms, PKC- $\gamma$ showed a reproducible upregulation in the DRG neurons and axons of peripheral sensory neurons of pain model rats. The expression of phosphorylated PKC- $\gamma$, the activated form of PKC- $\gamma$, in the DRG neurons and axons of peripheral sensory neurons of SNI rats was also detected in this study (unpublished data) and the result was similar to that of total PKC- $\gamma$. In general, both the total and phosphorylated PKC- $\gamma$ increased in the DRG neurons and axons of SNI rat model. Although the upregulation of PKC- $\gamma$ may modulate the sodium current and intracellular sodium channel trafficking, previous studies and our data provided no direct evidence to support the modulation of Nav1.5 mRNA and protein by PKC- $\gamma$. Combined with previous studies, we hypothesized that the activation of PKC may suppress the sodium current and activity, which in turn decreased the intracellular $\mathrm{Na}^{+}$and inhibit the activation of PKA, which may modulate the expression of Nav1.5 mRNA and protein. However, we admit that the mechanisms must be more complex than that we hypothesized. Future studies should make further investigation to reveal the detailed mechanisms underlying this phenomenon.

In conclusion, this study first confirmed the expression of both adult and neonatal Nav1.5 isoforms in the DRG neurons and axon of peripheral sensory neurons. In DRG neurons and axons of SNI pain model rats, the expression of both adult and neonatal Nav1.5 decreased by approximately a half in mRNA and protein level, however, the expression of PKC- $\gamma$ increased by $\sim 1$-fold. The upregulation of PKC- $\gamma$ may downregulate the expression of adult and neonatal Nav1.5 in SNI rat models and then further involve in the generation of neuropathic pain. Further study need to clarify the specific relation between the upregulation of PKC $-\gamma$ and the downregulation of Nav1.5 in pain models.

\section{Acknowledgements}

The authors would like to thank the staff of the Department of Neurosurgery of the First Hospital of China Medical University (Shenyang, China) for their technical assistance.

\section{Funding}

The present study was supported by the Liaoning Provincial Natural Science Foundation of China (grant no. 2014021097, awarded to JW) and the National Natural Science Foundation of China (grant no. 31100770, awarded to JW).

\section{Availability of data and material}

The datasets used and/or analyzed during the current study are available from the corresponding author on reasonable request.

\section{Authors' contributions}

GML and ZQDX designed the expriment. JW performed the experiment, and was a major contributor in writing the manu- 
script. YFB established the animal model of pain. SWO and YJW interpreted the data and proofread the manuscript. All authors read and approved the final manuscript.

\section{Ethics approval and consent to participate}

Not applicable.

\section{Consent for publication}

Not applicable.

\section{Competing interests}

The authors declare that they have no competing interests.

\section{References}

1. Liu M and Wood JN: The roles of sodium channels in nociception: Implications for mechanisms of neuropathic pain. Pain Med 12 (Suppl 3): S93-S99, 2011.

2. Cummins TR, Sheets PL and Waxman SG: The roles of sodium channels in nociception: Implications for mechanisms of pain. Pain 131: 243-257, 2007.

3. Catterall WA: From ionic currents to molecular mechanisms: The structure and function of voltage-gated sodium channels Neuron 26: 13-25, 2000.

4. Yu FH and Catterall WA: Overview of the voltage-gated sodium channel family. Genome Biol 4: 207, 2003.

5. Catterall WA: Voltage-gated sodium channels at 60: Structure, function and pathophysiology. J Physiol 590: 2577-2589, 2012

6. Laedermann CJ, Pertin M, Suter MR and Decosterd I: Voltage-gated sodium channel expression in mouse DRG after SNI leads to re-evaluation of projections of injured fibers. Mol Pain 10: 19, 2014.

7. Devor M: Sodium channels and mechanisms of neuropathic pain. J Pain 7 (Suppl 1): S3-S12, 2006.

8. Rook MB, Evers MM, Vos MA and Bierhuizen MF: Biology of cardiac sodium channel Nav1.5 expression. Cardiovasc Res 93 12-23, 2012.

9. Schroeter A, Walzik S, Blechschmidt S, Haufe V, Benndorf K and Zimmer T: Structure and function of splice variants of the cardiac voltage-gated sodium channel $\mathrm{Na}(\mathrm{v}) 1.5$. J Mol Cell Cardiol 49: 16-24, 2010.

10. Wang J, Ou SW, Wang YJ, Kameyama M, Kameyama A and Zong ZH: Analysis of four novel variants of Nav1.5/SCN5A cloned from the brain. Neurosci Res 64: 339-347, 2009.

11. Wang J, Ou SW, Wang YJ, Zong ZH, Lin L, Kameyama M and Kameyama A: New variants of Nav1.5/SCN5A encode $\mathrm{Na}^{+}$ channels in the brain. J Neurogenet 22: 57-75, 2008.

12. Xing D, Wang J, Ou S, Wang Y, Qiu B, Ding D, Guo F and Gao Q: Expression of neonatal Nav1.5 in human brain astrocytoma and its effect on proliferation, invasion and apoptosis of astrocytoma cells. Oncol Rep 31: 2692-2700, 2014.

13. Renganathan M, Dib-Hajj S and Waxman SG: Na(v)1.5 underlies the 'third TTX-R sodium current' in rat small DRG neurons Brain Res Mol Brain Res 106: 70-82, 2002.
14. Kerr NC, Holmes FE and Wynick D: Novel isoforms of the sodium channels Nav1.8 and Nav1.5 are produced by a conserved mechanism in mouse and rat. J Biol Chem 279: 24826-24833, 2004.

15. Kerr NC, Gao Z, Holmes FE, Hobson SA, Hancox JC, Wynick D and James AF: The sodium channel Nav1.5a is the predominant isoform expressed in adult mouse dorsal root ganglia and exhibits distinct inactivation properties from the full-length Nav1.5 channel. Mol Cell Neurosci 35: 283-291, 2007.

16. Ren CT, Li DM, Ou SW, Wang YJ, Lin Y,Zong ZH, Kameyama M and Kameyama A: Cloning and expression of the two new variants of Nav1.5/SCN5A in rat brain. Mol Cell Biochem 365: 139-148, 2012.

17. Hartmann HA, Colom LV, Sutherland ML and Noebels JL: Selective localization of cardiac SCN5A sodium channels in limbic regions of rat brain. Nat Neurosci 2: 593-595, 1999.

18. Wu L, Nishiyama K, Hollyfield JG and Wang Q: Localization of Nav1.5 sodium channel protein in the mouse brain. Neuroreport 13: 2547-2551, 2002

19. Frenz CT, Hansen A, Dupuis ND, Shultz N, Levinson SR, Finger TE and Dionne VE: NaV1.5 sodium channel window currents contribute to spontaneous firing in olfactory sensory neurons. J Neurophysiol 112: 1091-1104, 2014.

20. Onkal R, Mattis JH, Fraser SP, Diss JK, Shao D, Okuse K and Djamgoz MB: Alternative splicing of Nav1.5: An electrophysiological comparison of 'neonatal' and 'adult' isoforms and critical involvement of a lysine residue. J Cell Physiol 216: 716-726, 2008.

21. Decosterd I and Woolf CJ: Spared nerve injury: An animal model of persistent peripheral neuropathic pain. Pain 87: 149-158, 2000.

22. Ou SW, Kameyama A, Hao LY, Horiuchi M, Minobe E, Wang WY, Makita N and Kameyama M: Tetrodotoxin-resistant $\mathrm{Na}^{+}$channels in human neuroblastoma cells are encoded by new variants of Nav1.5/SCN5A. Eur J Neurosci 22: 793-801, 2005.

23. Fraser SP, Diss JK, Chioni AM, Mycielska ME, Pan H, Yamaci RF, Pani F, Siwy Z, Krasowska M, Grzywna Z, et al: Voltage-gated sodium channel expression and potentiation of human breast cancer metastasis. Clin Cancer Res 11: 5381-5389, 2005.

24. Wang W, Gu J, Li YQ and Tao YX: Are voltage-gated sodium channels on the dorsal root ganglion involved in the development of neuropathic pain? Mol Pain 7: 16, 2011.

25. Moldovan M, Alvarez S, Romer Rosberg M and Krarup C: Axonal voltage-gated ion channels as pharmacological targets for pain. Eur J Pharmacol 708: 105-112, 2013.

26. Numann R, Catterall WA and Scheuer T: Functional modulation of brain sodium channels by protein kinase $\mathrm{C}$ phosphorylation. Science 254: 115-118, 1991

27. Schreibmayer W: Isoform diversity and modulation of sodium channels by protein kinases. Cell Physiol Biochem 9: 187-200, 1999.

28. Grant AO and Wendt DJ: Block and modulation of cardiac $\mathrm{Na}^{+}$channels by antiarrhythmic drugs, neurotransmitters and hormones. Trends Pharmacol Sci 13: 352-358, 1992.

29. Tateyama M, Kurokawa J, Terrenoire C, Rivolta I and Kass RS: Stimulation of protein kinase $\mathrm{C}$ inhibits bursting in diseaselinked mutant human cardiac sodium channels. Circulation 107: 3216-3222, 2003.

30. Hallaq H, Wang DW, Kunic JD, George AL Jr, Wells KS and Murray KT: Activation of protein kinase C alters the intracellular distribution and mobility of cardiac $\mathrm{Na}^{+}$channels. Am J Physiol Heart Circ Physiol 302: H782-H789, 2012. 